\title{
CENTRAL IDEMPOTENTS IN GROUP RINGS ${ }^{1}$
}

\section{S. PASSMAN}

Let $K[G]$ denote the group ring of a finite group $G$ over a field $K$ of characteristic $p>0$. If $\alpha=\sum_{x \in G} a_{x} x \in K[G]$ we let the support of $\alpha$ be Supp $\alpha=\left\{x \in G \mid a_{x} \neq 0\right\}$. A well-known result of Osima [2, p. 178] gives the explicit form for the central idempotents in $K[G]$ and in particular shows that their support consists of $p^{\prime}$-elements of $G$. For most applications only the latter fact is needed. The proof of this result is character theoretic in nature and essentially requires lifting $K[G]$ to a group ring over some $p$-adic field. In this paper we give an elementary character-free proof of

Theorem. Let e be a central idempotent in $K[G]$. Then Supp $e$ consists of $p^{\prime}$-elements.

We require the following few facts:

(1) Let $P$ be a $p$-subgroup of $G$ and let $s$ denote the natural projection $s: K[G] \rightarrow K[\mathbf{C}(P)]$. Then $s$ induces a ring homomorphism, the Brauer homomorphism, from $\mathbf{Z}(K[G])$ into $\mathbf{Z}(K[\mathbf{C}(P)])[\mathbf{1}$, Satz 7A].

(2) Let $S$ denote the subspace of $K[G]$ spanned by all elements of the form $\alpha \beta-\beta \alpha$ with $\alpha, \beta \in K[G]$. Then for $\alpha_{1}, \alpha_{2}, \cdots, \alpha_{m} \in K[G]$ we have

$$
\left(\alpha_{1}+\alpha_{2}+\cdots+\alpha_{m}\right)^{p^{n}} \equiv \alpha_{1} p^{n}+\alpha_{2}^{p^{n}}+\cdots+\alpha_{m}{ }^{n} \quad(\bmod S)
$$

(see $[1$, Satz $3 \mathrm{~A}]$ ).

(3) Let $S$ be as above and let $x$ be a central element of $G$ of order a power of $p$. If $\alpha \in S$ then $x \notin \operatorname{Supp} \alpha$ (see [1, Satz 3B]).

Note that (3) above is merely the simple observation that if $x, y, z \in G$ and if $x$ is central in $K[G]$ then $x \notin \operatorname{Supp}(y z-z y)$.

We now proceed to prove the theorem. Suppose $z$ is an element of Supp $e$ which is not a $p^{\prime}$-element and write $z=x y=y x$ where $x \neq 1$ has order a power of $p$ and where $q$, the order of $y$, is prime to $p$. Let $P=\langle x\rangle$. Then by $(1), s(e)$ is a central idempotent in $K[\mathbf{C}(\mathrm{P})]$ and $z \in \operatorname{Supp} s(e)$. Thus it clearly suffices to assume that $x$ is central in $G$.

Choose integer $n$ with $p^{n} \geqq|G|$ and with $p^{n} \equiv 1(\bmod q)$ and set $\alpha=y^{-1} e$. If $\alpha=\sum_{g \in G} a_{g} g$ then by (2) $\alpha^{p^{n}} \equiv \sum\left(a_{g}\right)^{p^{n}} g^{p^{n}}(\bmod S)$.

Received by the editors February 7, 1969.

${ }^{1}$ Research supported in part by NSF Contract GP-8023. 
Now $p^{n} \geqq|G|$ so $g^{p^{n}}$ is a $p^{\prime}$-element and hence by (3), $x \notin \operatorname{Supp} \alpha^{p^{n}}$. On the other hand since $e$ is a central idempotent and since $p^{n} \equiv 1$ $(\bmod q)$ we have $\alpha^{p^{n}}=\left(y^{-1}\right)^{p^{n}} e^{p^{n}}=y^{-1} e=\alpha$. Since, by definition of $\alpha$, $x \in \operatorname{Supp} \alpha$, this is a contradiction and the result follows.

We remark that this proof holds for group rings $R[G]$ where $R$ is any commutative ring with 1 satisfying $p R=0$ and it yields the same result. In fact $R$ need not even be commutative since $1 \in R$ implies immediately $\mathbf{Z}(R[G]) \subseteq Z(R)[G]$. In addition this proof will also handle the twisted group rings $K^{t}[G]$ once the following simple observation is made.

(4) Let $Z$ be a central $p$-subgroup of $G$. Then $K^{t}[Z]$ is central in $K^{t}[G]$.

With this fact, (1) and (3) carry over easily to the twisted case.

The author would like to thank Professor Walter Feit for his helpful suggestions.

\section{REFERENCES}

1. R. Brauer, Zur Darstellungstheorie der Gruppen endlicher Ordnung. I, Math. Z. 63 (1956), 406-444.

2. M. Osima, Note on blocks of group characters, Math. J. Okayama Univ. 4 (1955), 175-188.

Yale University 